\title{
NUMERICAL SIMULATION OF THE UNSTEADY STATOR-ROTOR INTERACTION IN A LOW-SPEED AXIAL FAN INCLUDING EXPERIMENTAL VALIDATION
}

\author{
J.M. FERNÁNDEZ ORO ${ }^{\dagger},{ }^{*}$, K.M. ARGÜELLES DIAZ† \\ C. SANTOLARIA MORROS $\uparrow$, M. GALDO VEGA $\dagger$ \\ $\dagger$ Área de Mecánica de Fluidos, Universidad de Oviedo. \\ Campus de Viesques 33271, Gijón (Asturias), Spain
}

\begin{abstract}
The present study focuses on the analysis of the dynamic and periodic interaction between both fixed and rotating blade rows in a single axial stage turbomachine. The main goal is placed on the characterization of the unsteady flow structures involved in an axial flow blower of high reaction degree, relating them to working point variations and axial gap modifications.

A numerical 3D simulation of the complete stage is carried out, using a commercial code, FLUENT, that resolves the three-dimensional, unsteady turbulent flow inside the passages of a low-speed axial flow fan. For the closure of turbulence, both URANS modeling and LES techniques are used and compared. LES schemes are shown to be more accurate due to their good description of the largest eddy structures of the flow, but require careful near-wall treatment. Complementarily, an experimental facility was developed to obtain a physical description of the flow inside the machine. Both static and dynamic measurements were used in order to describe the interaction phenomena. A five-hole probe was employed for the static characterization, and hot wire anemometry techniques were used for the instantaneous response of the interaction.

The scope is based on the development of a methodology to understand the flow mechanisms related to the blade passing frequency in a single rotor-stator interaction.
\end{abstract}

\section{INTRODUCTION}

The unsteady behaviour of any turbomachine is based on the appearance of unsteady flow patterns due to the relative motion of blade surfaces. In fact, the existence of a steady relative flow, with a tangential velocity gradient in the blade passages, produces an unsteady flow when observed from an absolute frame of reference (Lyman, 1993). On the other hand, the existence of boundary layers associated to blade surfaces generates a lattice of wakes that will be mixed and transported throughout the machine, establishing new unsteady phenomena. All these unsteady features are also responsible for the energy exchange in the flow, so an accurate description of the unsteadiness may be very important when predicting the turbomachine performance. Therefore, there are two basic mechanisms related to the flow unsteadiness which have to be kept in mind. First, the row interaction, characterized as a potential effect due to the relative motion of the blades at the rotational speed, and second, the wake-blade interaction that introduces non-uniformities, caused by the incoming upstream wakes and the advection of the wake mixing (losses) into the mean passage-averaged flow.

In order to analyze these aspects of the flow inside an axial flow blower, both numerical and experimental techniques may be employed for that purpose. In this way, different numerical methods have been evolved over the years, from $2 \mathrm{D}$ axisymmetric models to complete 3D unsteady schemes, according to the geometric complexity of the machines and the capacities of computers. Nowadays, the number of blade passages configuring a machine is a critical issue when a URANS 3D simulation is attempted. In fact, steady 3D passage-to-passage averaged methods (Adamczyk, 1985), including deterministic stress modeling to take into account the periodic temporal effects of moving rows, have been recently developed as a useful tool for multistage configurations (Rhie et al., 1998; Van de Wall et al., 2000). Just when there are few rows and a limited number of blades, an unsteady procedure can be considered to solve the flow.

In the experimental investigation of unsteady flow fields within multistage turbomachinery, intrusive methods have always played an important role. Five-hole probes, rapid response transducers, hot wire or hot film measurements have generally been employed using single point measurements between blade rows. Recently, PIV systems are being introduced as a powerful technique to obtain blade-to-blade 2D images of the flow (Uzol et al., 2002; Chow et al., 2002), but unfortunately, these non-intrusive methods are more expensive and require special transparent characteristics in the test sections. However, hot wire anemometry can still provide complete information about the flow if used intensively.

In the present work, mechanisms involved in stator-rotor interaction, such as blade blockage and wake transport, will be shown. First of all, a brief description of the machine and the experimental facilities will be given. The experimental methodology will be overviewed with the definition of the measurement planes and the presentation of the hot wire anemometry procedures. This part will conclude by presenting the most relevant results. Next, a complete unsteady 3D numerical model will be introduced, detailing the basic parameters and the operating conditions. Also, an LES technique with near-wall modeling (WMLES) will be presented, showing better agreement with experimental data than classic

\footnotetext{
* Corresponding Author. E-mail: jesusfo@uniovi.es
} 
modeling of the Reynolds stresses, in spite of the coarse meshes that have been used. Finally, an exhaustive comparison between experimental and numerical results will be completed in order to validate the numerical simulation.

\section{EXPERIMENTAL SETUP}

Axial Flow Blower. The axial flow blower is composed of a single, stator-rotor stage. The stator has 13 inlet guide vanes (British $\mathrm{C} 1$ profile), while the rotor has 9 blades (NACA 65 profile). Basic geometrical parameters for both fixed and rotating blade rows at hub, midspan and tip sections are summarized in table 1.

\begin{tabular}{|c|c|c|c|c|c|c|}
\hline \multirow{3}{*}{ Radius (mm) } & \multicolumn{2}{|l|}{ HUB } & \multicolumn{2}{|c|}{ MIDSPAN } & \multicolumn{2}{|l|}{ TIP } \\
\hline & \multicolumn{2}{|l|}{190} & \multicolumn{2}{|l|}{300} & \multicolumn{2}{|l|}{410} \\
\hline & Stator & Rotor & Stator & Rotor & Stator & Rotor \\
\hline Chord length (mm) & 157.0 & 179.0 & 165.3 & 165.8 & 170.8 & 157.4 \\
\hline Solidity & 1.71 & 1.35 & 1.14 & 0.792 & 0.86 & 0.55 \\
\hline Stagger angle $\left({ }^{\circ}\right)$ & 18.11 & 48.64 & 12.97 & 59.70 & 10.77 & 66.45 \\
\hline Camber angle $\left({ }^{\circ}\right)$ & 37.1 & 13.76 & 27.8 & 7.42 & 23.3 & 5.74 \\
\hline Thickness-to-chord ratio (\%) & 3.0 & 12 & 3.0 & 9.43 & 3.0 & 8 \\
\hline Pitch (mm) & 91.8 & 132.6 & 145 & 209.4 & 198.1 & 286.2 \\
\hline Inlet flow angle $\left(^{\circ}\right)$ & 0.0 & 59.61 & 0.0 & 64.7 & 0.0 & 69.36 \\
\hline Outlet flow angle $\left(^{\circ}\right)$ & 31.17 & 47.73 & 20.73 & 60.07 & 15.68 & 67.16 \\
\hline
\end{tabular}

Table 1. Geometrical parameter of the axial fan stage.

The hub and tip diameters are 380 and $820 \mathrm{~mm}$ respectively, giving a hub-to-tip ratio of 0.46 . The stator-rotor nominal distance is $80 \mathrm{~mm}$ from IGV's trailing edge to blade's leading edge (approx. 50\% of the rotor blades chord at midspan), but the gap is modifiable through a set of internal annular rings that can be removed or added to decrease or increase the row separation. Figure 1 shows both stator and rotor profiles at the nominal distance for 10 radial positions.

Therefore, the lower stator-rotor distance can be fixed at $60 \mathrm{~mm}(37.5 \%$ of rotor chord), and the larger gap at $100 \mathrm{~mm}$ $(62.5 \%)$. For a rotational speed of $2400 \mathrm{rpm}(40 \mathrm{~Hz})$, the design flow rate is $18 \mathrm{~m}^{3} / \mathrm{s}$ (atmospheric conditions of $101 \mathrm{kPa}$ and $15^{\circ} \mathrm{C}$ at the intake), so the design flow coefficient is 0.42 (based on tip speed). The fan is driven by a $37 \mathrm{~kW}$ motor that is supplied with an industrial frequency converter.

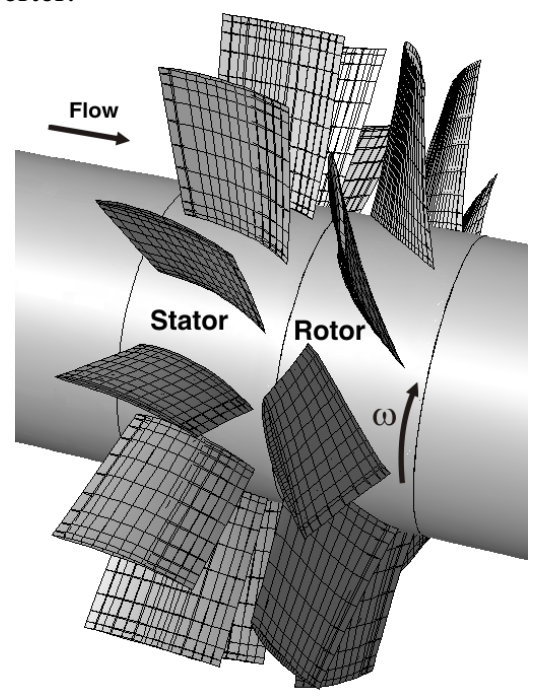

Figure 1. Single stage axial turbomachine

Test Facility. This test facility allows measurements at different transversal planes to be taken, including both upstream and downstream stage locations, as well as in the gap between the rows. Attached to the fan, a ducted part is used to modify the circuit characteristics, including a cone for flow rate control. A Venturi nozzle is coupled to the ducted fan, to obtain good measurements of the flow rate (according to the specifications of the British Standards Organization BS 848 for type A tests -free inlet, free outlet-, "Methods of Testing Performance", 1980). A long duct (16.5 meters) is enclosed between the fan bell-mouth inlet and the modifying cone to assure uniform flow at the fan intake (figure 2). 


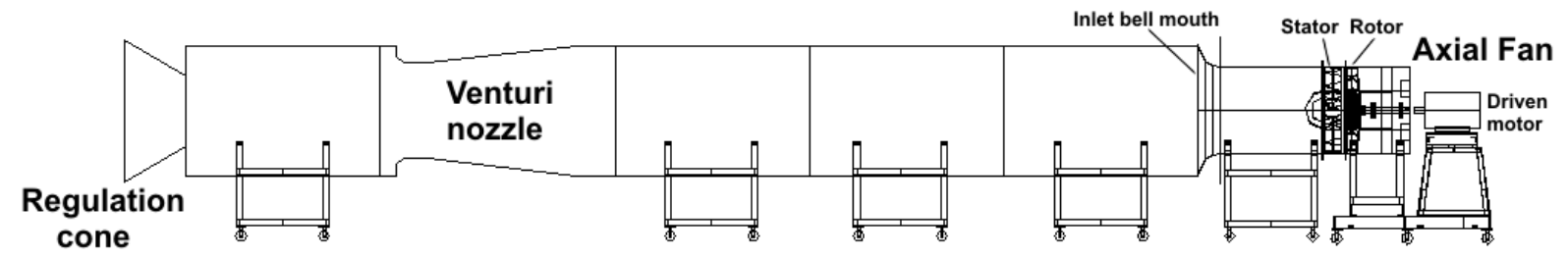

Figure 2. Sketch of the facility

Hot Wire Measuring Techniques. The experimental data has been obtained using dual hot wire anemometry. This technique gives a relatively high frequency response (15 to $20 \mathrm{kHz})$ to velocity fluctuations. This order of magnitude allows capturing flow patterns related to the blade passing frequency ( $360 \mathrm{~Hz}$ - nine blades rotating at $2400 \mathrm{rpm}$ ) and justifies the election of this measurement procedure. In addition, a trigger was introduced to match blade passing periods, so phase-averaged maps could be reconstructed later in the post-processing. The anemometric probe was composed of two tungsten filament wires of $5 \mu \mathrm{m} \mathrm{dia}$, in a $120^{\circ}$ crossed layout, with output signals connected to a TSI IFA-100 anemometer. The angular uncertainty was estimated in $2^{\circ}$, so relative uncertainty for velocity measurements is expected to be between 0.9 and $1.3 \%$. More details of the probe and the calibration setup can be found in Blanco et al. (1998).

Two different planes were chosen for the measurements (figure 3). The first plane (D) was placed $15 \mathrm{~mm}$ behind the stator trailing edge with the purpose of capturing flow effects between the rows, and a second plane (R) was located $50 \mathrm{~mm}$ downstream of the rotor trailing edge in order to analyze the outlet structures of the flow. Because of the periodic nonuniformity induced by the vanes, and considering that there are no clocking effects of previous stages, the measurements were limited to a circular sector, covering the whole span of the machine over the stator pitch. For a good spatial distribution, 15 single points were adopted along the radial coordinate, with a progressive separation according to an equal circumferential subtended area criterion. On the other hand, another 15 angular positions, every $2^{\circ}$, were used to complete the vane's passage in the tangential direction. As a result, two windows of 225 points for the upstream and downstream rotor flow were finally defined (figure 3, right).

A temporal resolution of 100 time instants per blade passing period has been considered (i.e. an acquired frequency of $36 \mathrm{kHz}$ ). Besides, another one hundred instantaneous realizations were recorded for each phase and location. This means that data of 100 rotor passages at every single measurement point have been acquired, so ten thousand values of both axial and tangential velocity were captured for the $15 \times 15$ sector grid. Using these instantaneous measurements, phase-averaged velocity maps can be obtained by averaging the hundred passages, thus removing the turbulent flow phenomena, according to:

$$
\bar{u}_{i}(r, \theta, \phi)=\left.\frac{1}{N} \sum_{k=1}^{N} u_{i}(r, \theta, \varphi)\right|_{k} ; \quad \varphi=\phi+\frac{2 \pi}{B}(k-1)
$$

where $\mathrm{N}=100$ is the number of instantaneous window maps and for each phase, $r$ and $\theta$ are the radial and tangential coordinates, respectively, and $\phi$ is the phase angle of the rotor passage. $B$ is the number of blades and $\varphi$ computes the total angular displacement. The subscript $i$ takes values $(a x)$ and $(t g)$ representing axial and tangential velocities.

This phase-averaged flow at 100 different rotor phases (every $0.4^{\circ}$ of rotor position, which covers the entire rotor passage) allows the passage-averaged flow field to be obtained (Sentker and Reiss, 1998, 2000). Taking into account that the present phase-averaged flow is an ensemble average of $\mathrm{N}=100$ instantaneous realizations, the passage-averaged flow field is, essentially, calculated by time-averaging of the ensemble averaged parameters. Then,

$$
\tilde{u}_{i}(r, \theta)=\left.\frac{1}{M} \sum_{k=1}^{M} \bar{u}_{i}(r, \theta, \phi)\right|_{k}
$$

where $M=100$ is the total number of phase-averaged realizations, covering an entire passage. Notice that the phase is removed for the dotted velocities, so time-averaged variables are phase-rotor independent values (steady maps).
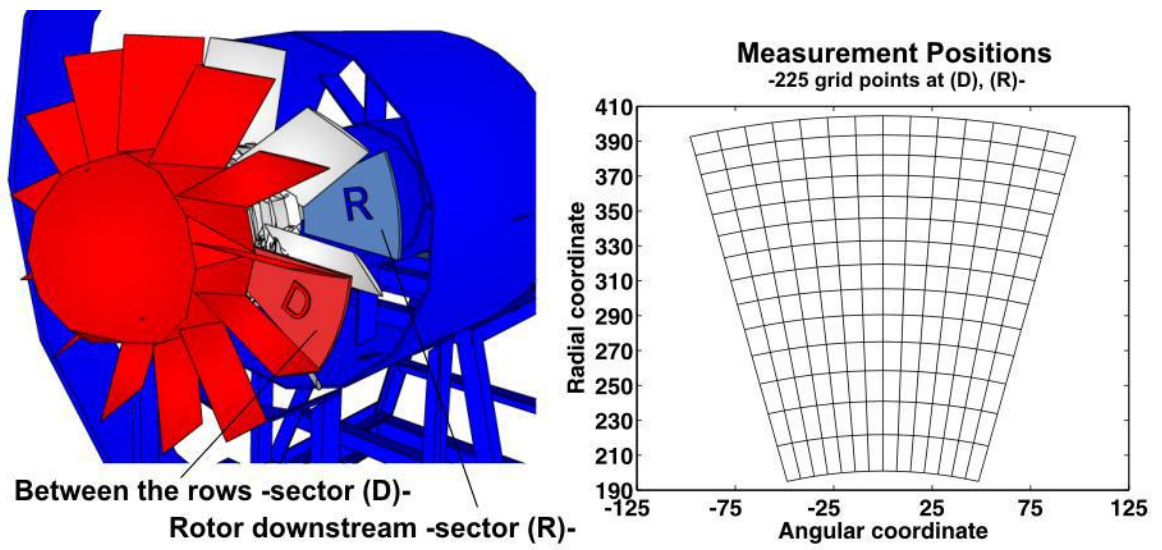

Figure 3. Measurement locations. 


\section{EXPERIMENTAL RESULTS}

Both time-averaged and instantaneous results will be shown below. The time-averaged (passage-averaged) flow represents in a unique map the mean contribution of the unsteady flow structures, while instantaneous maps (phaseaveraged flow) require of different snapshots to be piled up in order to provide the complete evolution of the unsteadiness.

In addition, both absolute and relative reference frames have been considered for the discussion. The velocity fields in the relative frame were retrieved relocating the measured values according to the temporal lag that fulfills time periodicity. In essence, it is necessary to convert the fixed coordinates of the stator into the moving coordinate system of the rotor, following a rotation of coordinates expressed as $\theta_{r e l}=\theta_{a b s}-\Omega t$. On the other hand, a typical convention is to adimensionalize the particular time step of every snapshot with the rotor blade passing period, $t / T_{R}$, when observed in the absolute frame of reference. Alternatively, the stator vane passing period, $T_{S}$, is employed when presenting the relative frame of reference. This is because when using the fixed reference frame, the periodicity of the flow patterns is imposed by the rotor blade passing period over the vane passage, whereas for the relative frame of reference, the periodicity is imposed by the stator vane passing period over the rotor passage (in this case, the guide vanes are "observed" rotating in the opposite direction). Therefore, the aspect ratio of both reference frames differs for both sector graphs (D and R). Finally, for a better representation in both reference frames, the data has been duplicated tangentially, so two stator pitches $\left(55^{\circ}\right)$ are always drawn in the fixed reference frame (i.e. figure $5 \mathrm{~b}$ ), while two rotor pitches $\left(80^{\circ}\right)$ are always drawn in the moving reference frame (i.e. figure $4 b$ ).

Stator-Rotor Wake Generation. A first approach to characterize the flow pattern inside the turbomachine is shown in figures 4 and 5 .
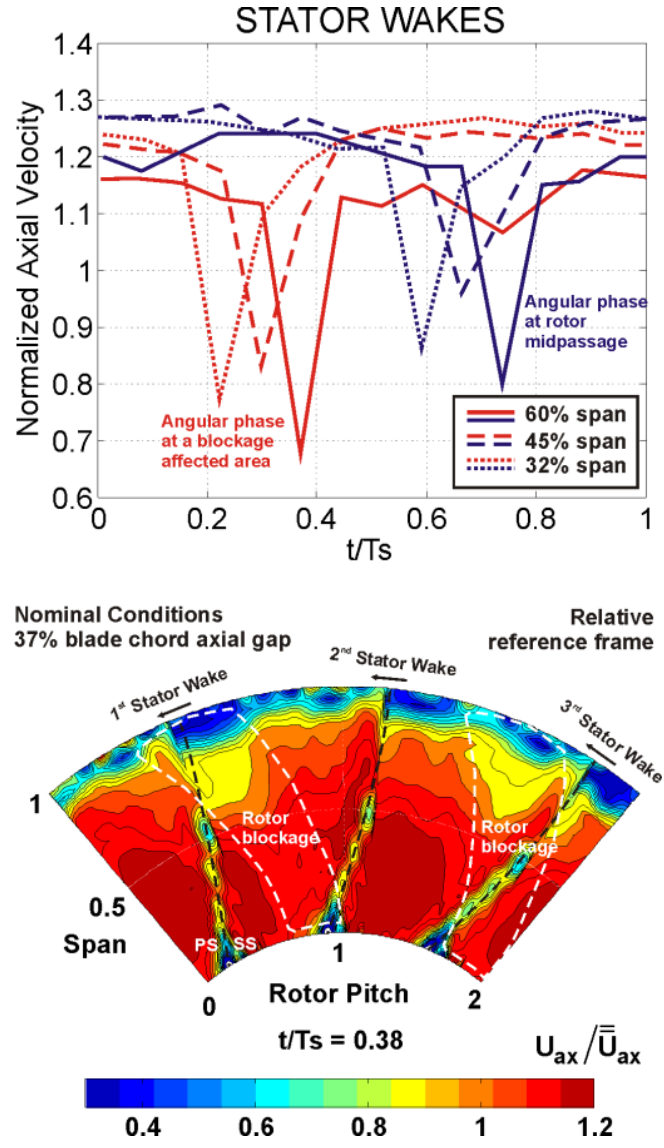

Figure 4. Stator wakes.
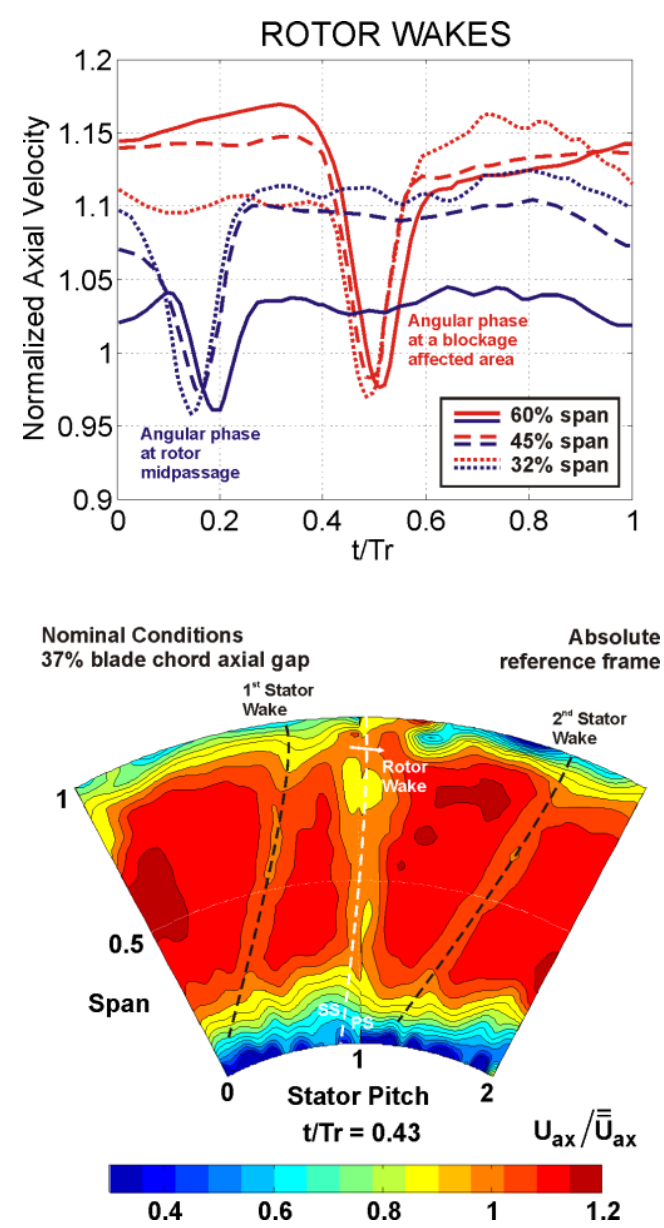

Figure 5. Rotor wakes.

Figure $4 \mathrm{~b}$ shows the normalized axial velocity in the sector between the rows (D), viewed from the moving reference frame. The axial gap is equal to $37 \%$ of the blade chord at midspan and the operating point is the nominal flow rate. The normalization is calculated with the overall mean value of the axial velocity. Notice that it is an instantaneous result, corresponding to $t / T_{S}=0.38$. To obtain these velocities in the rotor frame of reference a repositioning procedure (phaselagged matching) is used with the absolute velocity maps. As a result, three stator wakes are observed rotating in the opposite direction to that of the rotor blades in the fixed frame, viewed from the machine discharge. The stator wakes are clearly visible, although the angular discretization was rather too imprecise to capture them smoothly. In addition, the effect of the rotor blades blockage induces a low-velocity region (dashed-line region), breaking the tangential uniformity in the passage distribution. Therefore, two different flow structures are identified: the stator wakes and the rotor blockage. Near 
the tip, there is an important reduction of the axial velocity caused by the rotor blockage. This reduction is more extreme when the stator wakes occur in those angular phases. Figure $4 \mathrm{a}$ has been introduced to illustrate this interaction between the wakes and the blockage. In the plot, the evolution of the normalized axial velocity is represented at three different spanwise locations (32\%, $45 \%$ and $60 \%$ of the span) for two angular phases (red and blue). The angular phase marked in red is placed in the blockage-affected zone of the passage (dashed-line region in figure 4b), while the blue one is placed in the zone with maximum velocity values. The shape of the stator wakes is very sharp for both angular phases, reaching $40 \%$ decay in the velocity value, but the deficits in the red angular phase are more pronounced than those in the blue one.

Figure $5 \mathrm{~b}$ shows the shape of the rotor wakes in the absolute frame of reference. The figure introduces a snapshot $\left(t / T_{R}\right.$ $=0.43$ ) of the normalized axial velocity in the sector $(\mathrm{R})$, downstream of the rotor, for an axial gap of $37 \%$ of blade chord and design working point. The rotor wakes present a lower shape factor than those at the stator, with maximum deficit values of just 15-20\% (see Fernández Oro et al., 2007, for more details). The most important feature is the presence of the stator wakes in the measurement plane downstream of the rotor. Although they have been transported and partially mixed out with the mean flow patterns throughout the rotor passages, the stator-rotor distance is too small to produce a complete mixing-out process of the stator wakes. Therefore, despite being stretched in the rotor passage, they are surprisingly visible at the machine discharge. Once again, a clear unsteady mechanism takes place through the periodic interaction of both rotor and stator wakes. Figure 5a is introduced to illustrate this phenomena. The plot shows the evolution of the normalized axial velocity at the same spanwise locations as before for two angular phases (red and blue). The blue angular phase is located in the same angular position as the stator wakes. As expected, the axial velocity presents a low mean value (when compared to red curves, placed in zones unaffected by the stator wakes). Besides, the deficit produced by the rotor wakes is less deep. Quantitatively, it can be estimated as 30-60\% lesser, depending on the spanwise locations (it suggests a relationship with the aerodynamic load of the blades).

Passage-Averaged Flow. The passage-averaged flow shows the impact of the unsteady structures on the mean time flow patterns. To analyze the influence of the axial gap and the mass flow rate conditions, the normalized axial velocity has been represented between the rows in figure 6 in the absolute frame of reference. On one hand, two axial gap configurations were tested: $50 \%$ and $37 \%$ of blade chord at midspan. In figure 6 , the left column shows results for the larger axial gap, while the right shows results for the lower gap. On the other hand, three different flow rates were considered: nominal flow rate $\left(\mathrm{Q}_{\mathrm{n}}=16.5 \mathrm{~m}^{3} / \mathrm{s}\right)$, partial load $\left(85 \% \mathrm{Q}_{\mathrm{n}}\right)$ and partial load near stall $\left(70 \% \mathrm{Q}_{\mathrm{n}}\right)$. In the figure, every column includes the map distributions for these three flow conditions.
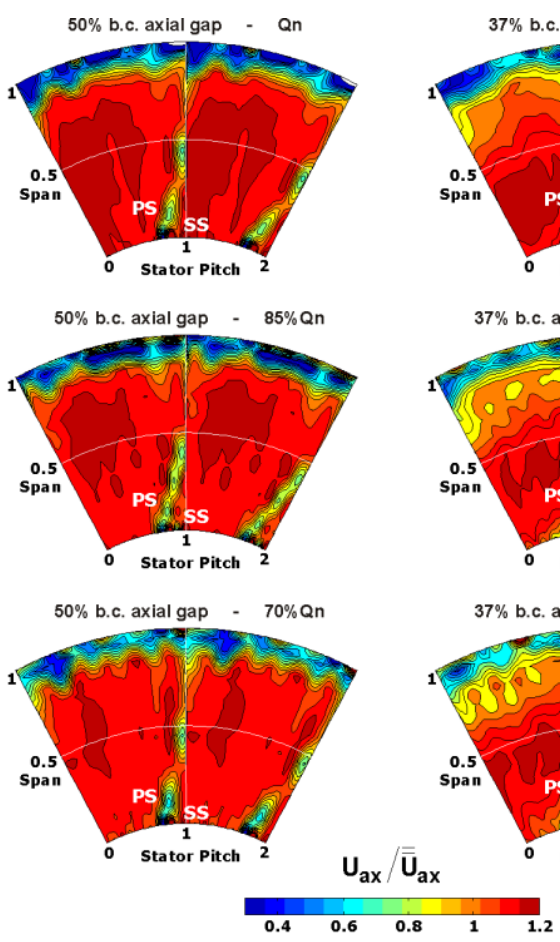

sector (D), between the rows (Absolute frame of reference)

Passage-averaged Axial Velocity
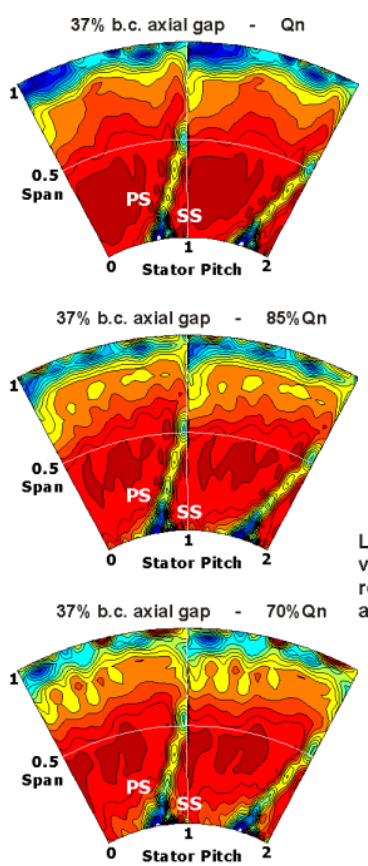

velocity region the tip
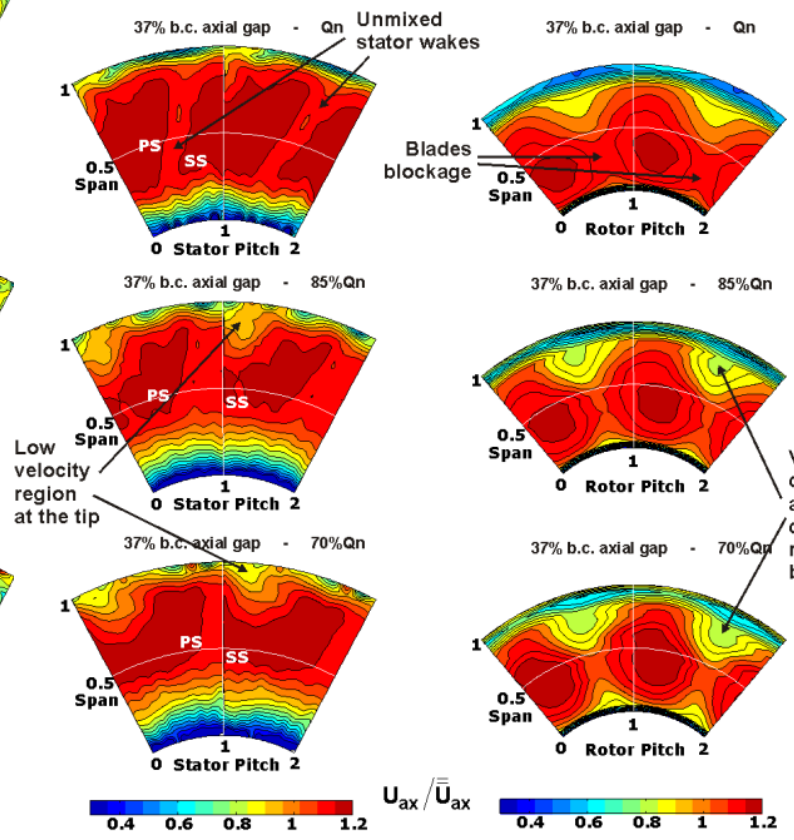

-sector $(R)$, rotor downstream(Absolute frame of reference)

Passage-averaged Axial Velocity

Figure 6. Passage-averaged velocity between the rows

Figure 7. Passage-averaged velocity rotor downstream

The results show that major differences are due to modifications in the axial gap distance. A good tangential uniformity can be observed in all the cases, only broken by the stator wakes discontinuities. Nevertheless, the lower axial gap produces a clear spanwise redistribution of the velocity map, blocking the flow pattern from midspan to tip locations, so the flow is concentrated near the hub. By decreasing the mass flow rate, the differences between low-velocity tip zones and high-velocity hub zones are wider. In addition, the intensity of the stator wakes deficit is greater in the case of reduced 
axial gap, because of the inherent non-uniform flow pattern. When the flow is more uniform, the wakes mixing out is more evident.

Mass flow rate influence is expected to be more critical in the flow features downstream of the rotor. Effectively, a reduction in the flow velocity could hardly affect stator flow uniformity, but rotor inlet conditions and blade loads could vary dramatically. The left column in figure 7 shows the outflow conditions in the case of $37 \%$ of blade chord axial gap. The normalized axial velocity is represented once again at $\mathrm{Q}_{\mathrm{n}}, 85 \% \mathrm{Q}_{\mathrm{n}}$ and $70 \% \mathrm{Q}_{\mathrm{n}}$. The averaging procedure has filtered blade passing effects, so just partially mixed-out stator wakes are visible. At partial load, the shape of these wakes becomes more diffuse, but on the other hand, a low-velocity zone at the tip grows. Since this low-velocity region has not been smeared by passage-averaging, then this phenomenon is not linked to the blade passing frequency (BPF), but related somehow to stator outflow conditions. Therefore, the figures indicate that this flow pattern is generated by the stator vanes. Complementarily, the right column in figure 7 is introduced to explain the propagation and the generation of this flow feature. This column shows the normalized axial velocity between the rows when observed in the moving reference frame. In the relative frame of reference, stator wakes are filtered so just the effect of rotor blockage is revealed. At nominal flow rate, a velocity deficit is generated at the tip regions for every blade; but at partial load, the difference between this tip deficit and the midspan high-velocity zone becomes more severe. These O-shape low-velocity structures are responsible for the existence of the low-velocity tangential path between the rows, when viewed in the absolute frame of reference in figure 6. These patterns, which travel within the rotor blades, interact with the stator wakes, thus increasing the velocity deficits when both effects intersect (especially at the vanes' suction side). Nevertheless, the tangential deficit at the tip is active although the actual rotor wake has passed away or the next wake is about to pass. Consequently, in the left column of figure 7, at lower axial gap, despite the averaging technique, this effect appears associated with the suction side of the guide vanes. This discussion illustrates how some unsteady characteristics of the stator-rotor interaction can be explained by taking into account both fixed and moving reference frames. Particularly, the generation of this phenomenon is explained in the stator frame, while its propagation was shown in the complementary rotor frame. In the literature, similar massive decays of axial velocity due to large flow blockages at the tip have been also observed by Jang et al. (2005) for this kind of NACA-65 profiles in an isolated rotor, especially close to near-stall conditions.

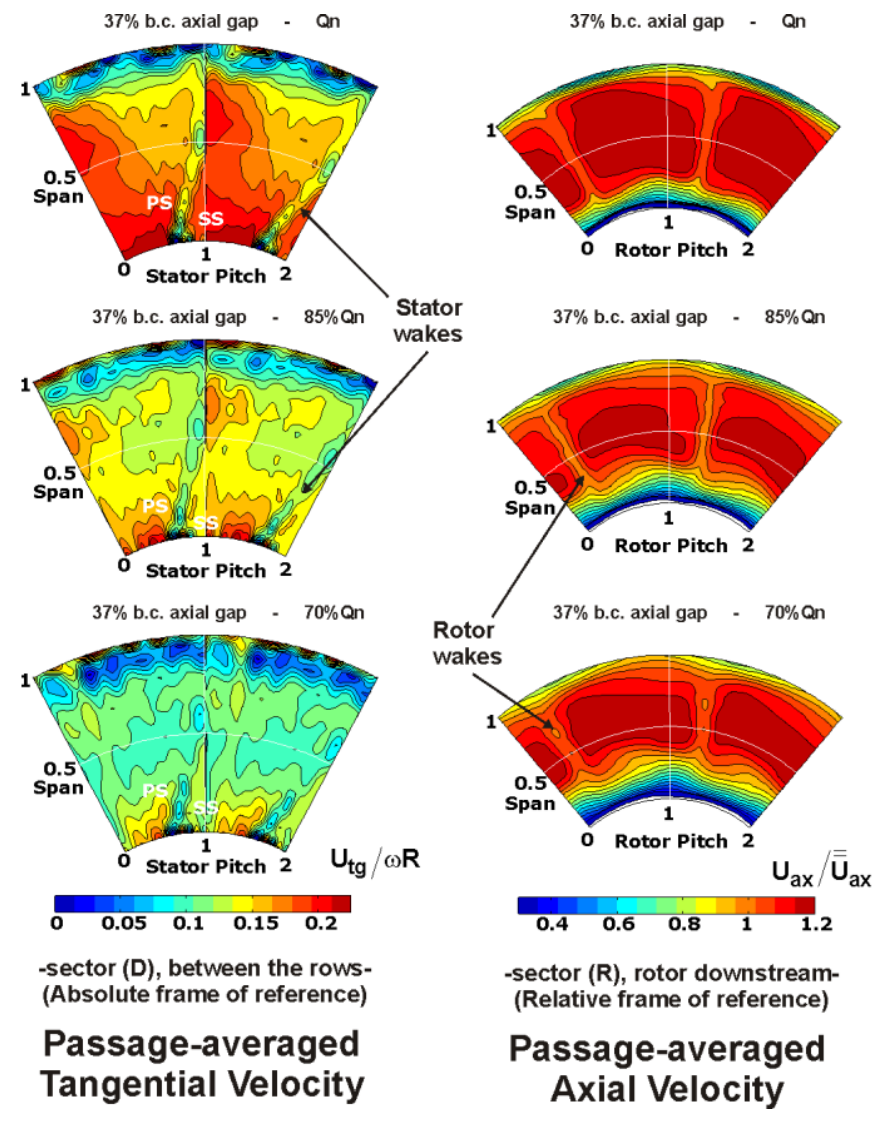

Figure 8. Passage-averaged axial and tangential velocities.

The left column in figure 8 shows the tangential velocity in sector (D), between the rows, normalized by the tip blade velocity. A quite uniform gradient of tangential velocity decreases spanwise, matching with the geometrical parameters of the vanes summarized in table 1. Also, and superimposed to this background behaviour, two different zones with high values appear in the representation. The first is placed in the hub region, as a consequence of the time-averaging of the rotor blockage. The second is observed at the tip zone of the vanes suction side, amplified by the dynamic interaction discussed before. This structure is identically developed at partial load, with maximum values perfectly recognizable at same locations. Finally, the right column of figure 8 is introduced to show the progressive growth of the rotor wakes as the 
mass flow rate decreases. As expected, a major flow separation produces thicker wakes at $70 \%$ Qn, as well as an enlargement of the hub boundary layer.

Phase-Averaged Flow. The unsteady effects of rotor-wake and wake-wake interaction will be considered in this section. Thus, figure 9 shows instantaneous phase-averaged maps at five intermediate snapshots in both stator and rotor frames of reference. The left column displays the normalized axial velocity in sector (D), for the absolute frame of reference, in five different instants of the blade passing period: $t / T_{R}=0.0,0.2,0.4,0.6$ and 0.8 . The case of a $37 \%$ blade chord axial gap, at partial load $\left(85 \% \mathrm{Q}_{\mathrm{n}}\right)$, is used to illustrate the radial interaction that is established between the stator wakes and the rotating blockage effect of the blades. The deficit in the stator wakes is clearly increased when the blockage passes along the wake path. Observe the differences in the central wake intensity at $t / T_{R}=0.0$ and $t / T_{R}=0.4$. Because of the relative transverse position of vane's trailing edges and blade's leading edges, a radial migration of wake-rotor interaction is established during the rotor passing period. Notice how similar trends were previously shown in the graph of figure 4 . In addition, the evolution of the tip low-velocity region can be noticed moving tangentially with the rotor, and how it is interacting with the suction side of the stator vanes.

In the case of nominal flow rate, $37 \%$ of blade chord axial gap, some conclusions can be reached from the normalized relative axial velocity maps in the right column of figure 9. Oscillations in the rotor wakes can be guessed at tip zones when they intersect with unmixed stator wakes (compare the relative position of the rotor wake respect to the vertical dashed line overtime). Aditionally, the relative counter-clockwise motion of stator wakes thickens the rotor ones, especially at the tip $\left(t / T_{S}=0.0\right.$-right rotor wake- and $t / T_{S}=0.6$-left rotor wake). This time, the trend that was pointed out in the graph of figure 5 is now confirmed, showing a wake-wake interaction as an unsteady periodic flow pattern.

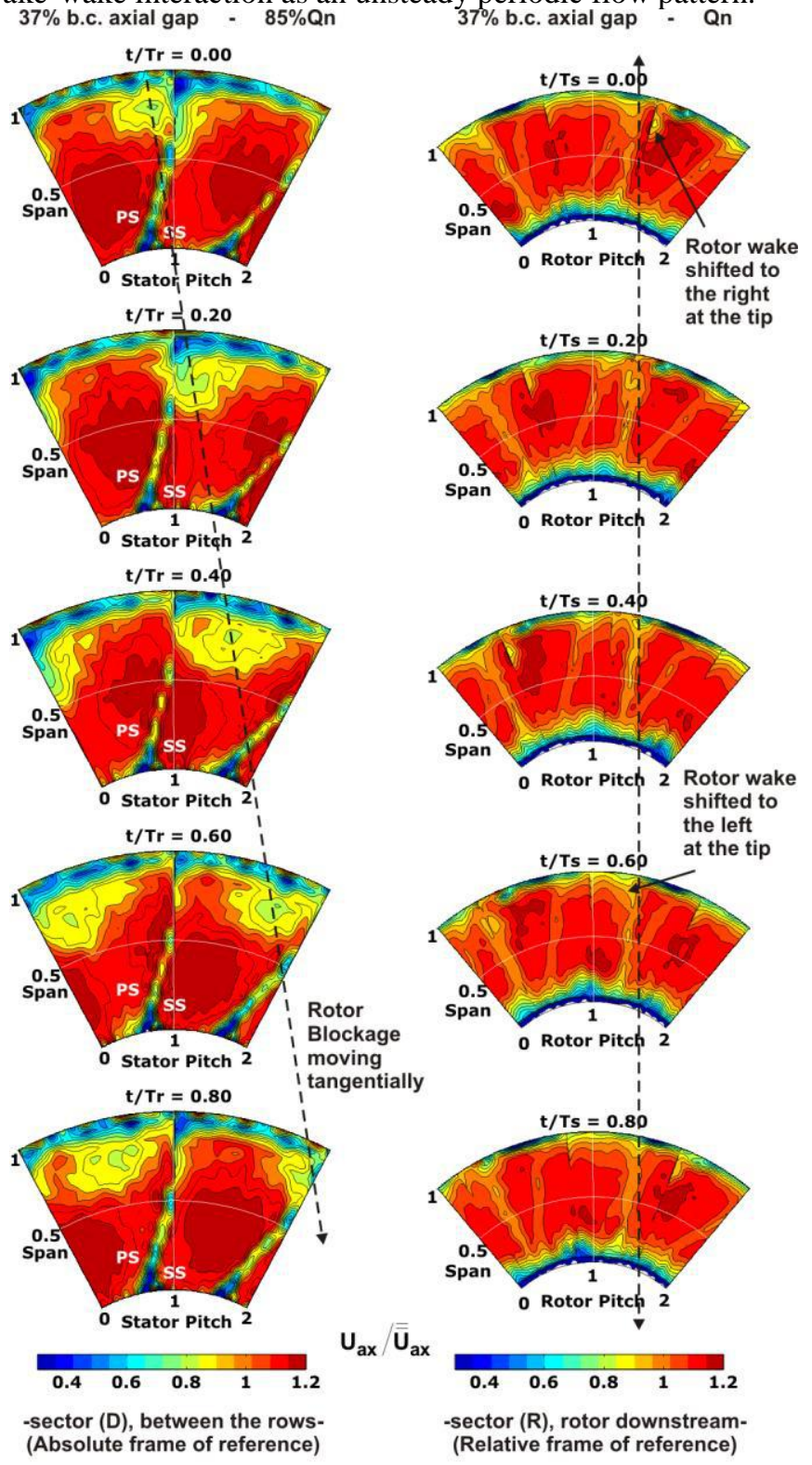

Phase-averaged Axial Velocity

Figure 9. Phase-averaged (unsteady) velocity. 
To conclude, figure 10 shows a space-time three-dimensional contour plot of the normalized axial velocity at the midspan. The evolution at all the angular positions is shown for the nominal flow rate condition, with $37 \%$ of blade chord axial gap. Due to the rotor motion, the rotor wake exhibits a transversal orientation for the time axis, while stator wakes appear in the same angular coordinate all the time. This figure is really an extension of the plot in figure 5, which summarised this evolution for just two different angular phases: at $18^{\circ}$ (over the stator wake - blue curves) and $4^{\circ}$ (over stator midpassage - red curves).

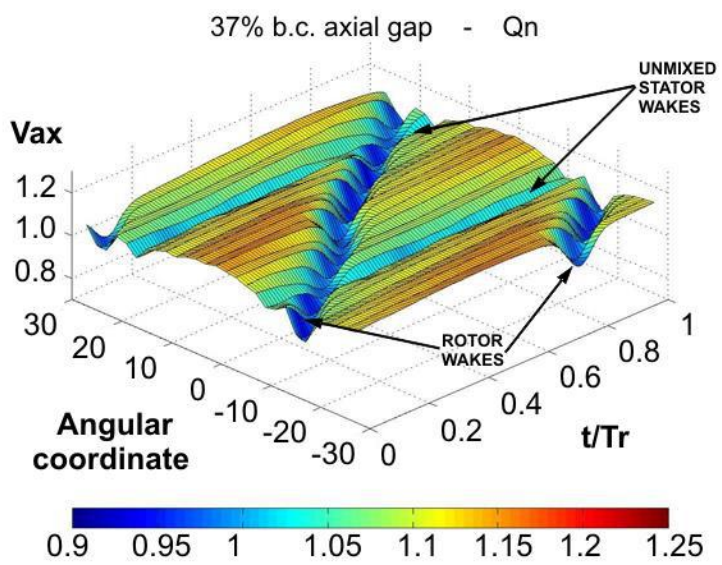

Figure 10. Unmixed stator wakes.

\section{NUMERICAL METHODOLOGY}

Unsteady 3D Numerical Simulation. The commercial CFD software FLUENT v6.1 was used to solve the NavierStokes set of equations. The code, unsteady, viscous and three-dimensional, introduces a volume finite method with an explicit, second-order accurate scheme for the temporal discretization. A SIMPLE algorithm is chosen for the pressure and velocity coupling, and central differencing discretizations have been used for convection and diffusion terms.

A compatible preprocessor, GAMBIT v2.0, was used to develop both geometries and meshes. No simplifications for the relative number of blades (9) and vanes (13) were adopted, so a full 3D annulus grid of the stage was implemented with 22 total passages and aprox. 2 million cells for the whole domain (figure 11). The final discretization was generated according to maximum computational capacities, composed of an $8 \mathrm{PC}$ cluster, $2.6 \mathrm{GHz}$, parallelized with a Gigabit connection set. As a result, a [35x100x25] O-type grid distribution was introduced for every passage, thus increasing the grid resolution near the surfaces. On the contrary, in order to prevent excessive grid size discontinuities in the tip region, clearance gridding was not implemented in the present model. It is well-known in the literature (see for instante Hirsch, 2007) that severe grid stretching reduces the accuracy of the mesh into first order, leading to more numerical diffusion and poorer solutions. Since non-uniform grids are currently a must if tip gridding is attempted with the available computational resources, the clearance was not finally modelled to preserve second-order accuracy. Furthermore, attempt LES calculations on the tip region of multistage axial turbomachinery is non realistic with the present limitations. As a consequence, the model was built up with a rotor casing that rotates along with the blades domain, introducing a 25-node radial distribution that guarantees a correct description of the spanwise gradients. Moreover, the moderate concentration of cell nodes towards hub and tip sections was carefully designed to obtain accurate $\mathrm{y}^{+}$values (see next section) throughout the whole passage span. Figure 11 also shows that cells were extruded spanwise from hub to tip, even in the rotor passages, where blade twisting provokes high skewed elements.

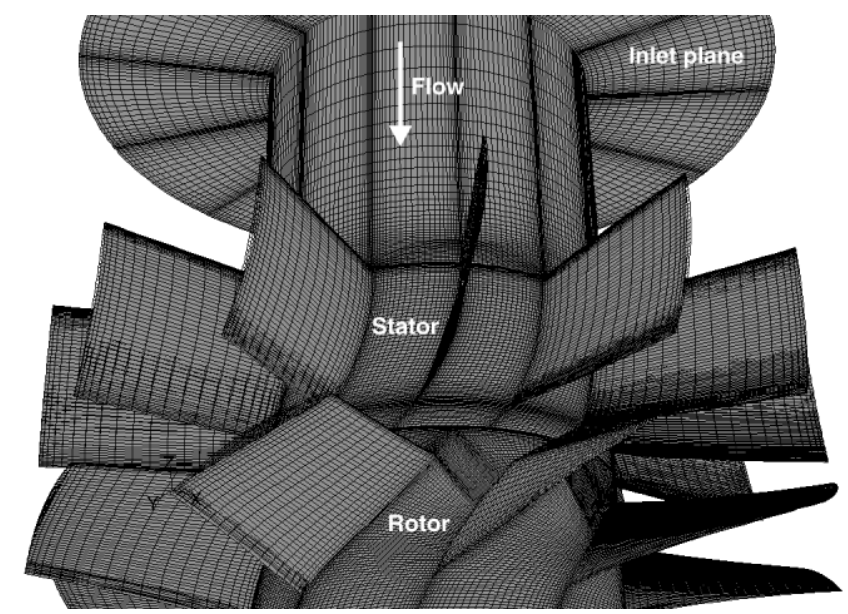

Figure 11. Numerical domain and mesh. 
Using a "sliding-mesh" technique, the unsteady simulation provides a temporal description of the flow variables throughout the stage. Thereby, the rotor zone is forced to rotate around the axis at $2400 \mathrm{rpm}$. In order to obtain a good resolution for the velocity fields, $468[13 \times 9 \times 4]$ intervals per rotor revolution were adopted (52 per rotor passage), so the time step was fixed at $5.3418 \times 10^{-5} \mathrm{~s}$. Notice that the total number of intervals per rotor passage in the numerical simulation (52) is calculated in the same way as the total number of phase-averaged realizations covering a passage in the experiments (100). With the CFD code, it is also possible to store the flow variables for each interval, in order to use them in a forward post-processing (i.e. passage-averaging).

WMLES vs URANS. The incompressible Navier-Stokes equations are always closed using a turbulence modeling in any numerical simulation. Basically, this procedure is analogous to the ensemble-averaging technique introduced for the experimental measurements. As a commercial code, FLUENT provides a wide range of turbulent models, from the classical Reynolds-averaged schemes (RANS) to large eddy simulation (LES) techniques. In this study, both RANS modeling and LES techniques were tested to find the most accurate scheme for the simulation.

1) An unsteady RANS (URANS) model was first introduced. This scheme models all turbulence scales, solving ensemble-averaged Navier-Stokes equations. RANS modeling offers different possibilities (Spalart-Allmaras, k- $\varepsilon$, k- $\omega$, RSM) according to increasing complexity. In this case, a Reynolds Stress Model (RSM) has been chosen because of its suitable predictions for rotating flow passages. Effects of curvature, swirl and rotation are directly accounted for in the transport equation, and the turbulence is considered anisotropic. Moreover, recent investigations by Uzol et al. (2007) in a 2-stage, low-speed axial turbomachine have demonstrated the propensity of the k- $\varepsilon$ model (also of applicance to similar eddy-viscosity models like k- $\omega$ ) to overestimate turbulence production near the stagnation points when compared to RSM results, which provides more reasonable turbulence levels (see also Fernández Oro et al., 2009, for additional details of the RSM model implementation in an isolated rotor of an axial jet fan).

Near-wall modeling has been introduced with a standard logarithmic wall function, so wall-adjacent cells are not placed in the buffer layer $\left(\mathrm{y}^{+} \sim 30\right)$. Outer region and log-law layer are properly resolved by the RSM scheme near the walls. Considering a Reynolds number based on tip characteristics $\left(\operatorname{Re}_{\mathrm{Ch}}=12 \times 10^{5}\right)$, the first grid point is placed at $0.2 \mathrm{~mm}$ from the blade surfaces, with a growth factor ratio of 1.3. As a result, a $\mathrm{y}^{+}$value of 60 wall units was fixed at both suction and pressure side of the blades.

2) Using URANS results as initial conditions, an LES technique was attempted to resolve the larger eddies. In this case, the governing equations are grid-filtered so subgrid-scale stress terms are introduced into the model. The subgrid-scale stresses are unknown and require modeling. Thus, a Smagorinsky-Lilly model has been used, with a default value of $C_{S}=$ 0.1 . The requirement for a well-resolved LES in the near-wall region expressed in wall units is approximately $\Delta \mathrm{x}^{+} \sim 100$ (streamwise) and $\Delta \mathrm{z}^{+} \sim 20$ (spanwise). Also, the near-wall grid spacing should be about one wall unit in the wall-normal direction, $\Delta y^{+} \sim 1$ (Davidson and Dahlström, 2005). These grid requirements are not affordable for actual industrial computational capacities in the case of a full annulus 3D domain. It can be said that wall-bounded flows at high Reynolds numbers need computational resources prohibitively large for pure LES algorithms.

To avoid the extreme requirement of near-wall grid resolution, the LES scheme is used including near-wall modeling below the inner part of the logarithmic region (between 30 to 60 wall units away from the wall). Then, the near-wall region is sufficiently resolved and hence the near-wall model is automatically applied by FLUENT in the LES simulations, resulting in a wall-modeled large eddy simulation (WMLES). This approach allows the first grid point to be located in the $\log$ layer, but the vortical structures in both viscous and buffer regions are not resolved, and therefore coarse meshes can be used in all the directions $\left(\Delta \mathrm{x}^{+} \sim 100-600, \Delta \mathrm{z}^{+} \sim 100-300\right)$. This means that the interaction between the modeled, near-wall region, and the resolved, outer region, is weak, assuming that only the Reynolds shear stresses are significant. Thus, the stress at the wall is computed in terms of the velocity at the first inner point, and the dynamics of the wall layer is considered to be more universal (Piomelli et al., 2006).

Time step size is another restrictive parameter for LES techniques. Generally, it should be small enough to resolve the time-scale of the smallest resolved eddies. According to the mesh density, the order of magnitude of these eddies is that of the wakes' shear layer at the blade's trailing edge. Considering the through-flow velocity as a representative value, at least one time step in the range of $10^{-5} \mathrm{~s}$ must be fixed to capture the eddies shedding.

Obviously, numerical simulations with LES algorithms require finer meshes and lower time steps if complete vortex shedding needs to be described. Otherwise, just larger eddies are captured and resolved. However, an initial approach to LES techniques has been explored in this study, resulting in a description of the unsteady stator-rotor interaction that follows previous experimental data. In this case, WMLES allows the calculation to reflect the instability of the shear layer and the development of the coherent structures in the wake. In addition, more accurate prediction of the unsteady forces and of the sound emission can be obtained when compared to steady or unsteady pure RANS methods (Piomelli et al., 2006). Effectively, in this study the URANS solution has demonstrated notable numerical diffusion and poor prediction of the aero-acoustic noise than WMLES computations (Argüelles et al., 2009).

Figure 12 is introduced to illustrate the best performance of WMLES modeling. Reproducing similar flow features of that in figure 10, a comparison between WMLES and RSM shows a better description of the wakes shape when WMLES is applied. Besides, the presence of unmixed stator wakes is captured in a similar fashion to that exhibited experimentally. On the contrary, classic Reynolds Stresses Models present much more diffused rotor wakes, due to a major numerical 
diffusion. Also, stator wakes have been completely filtered in the RSM, pointing to an overestimation in diffusive mechanisms.
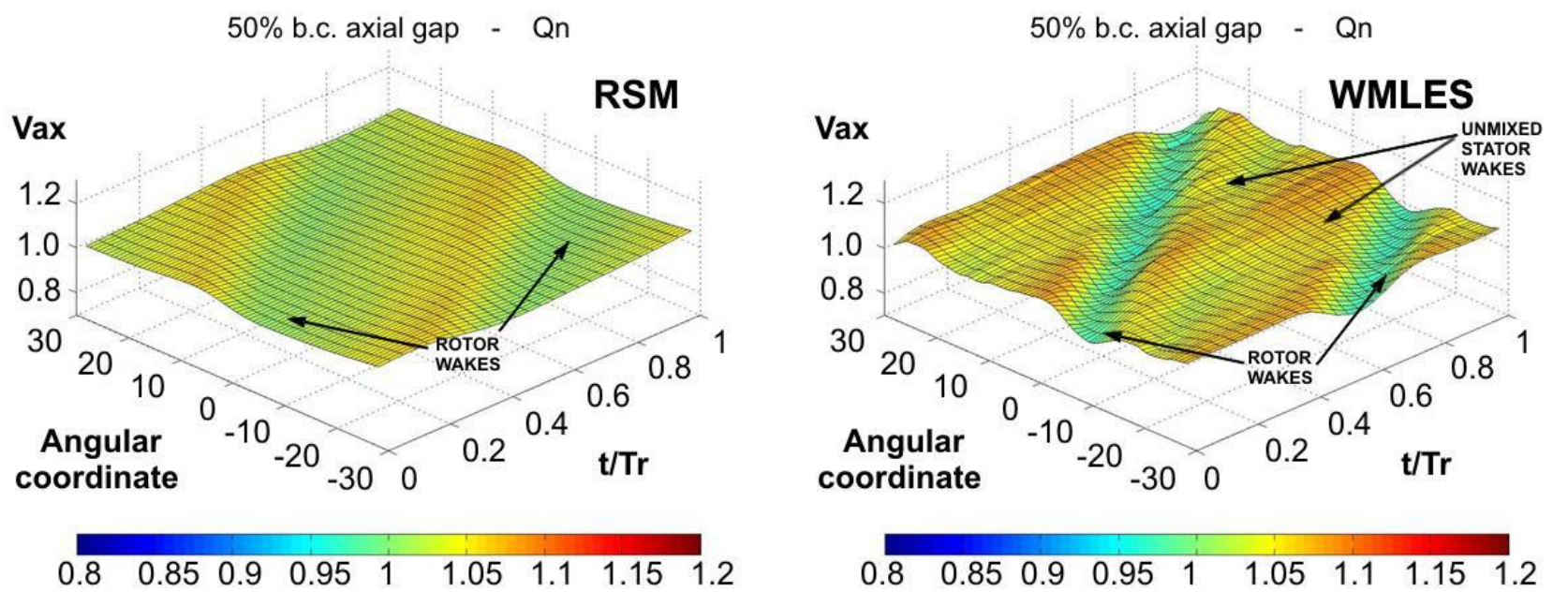

Figure 12. Numerical predictions (comparison)

BC's and overall convergence. Finally, both pressure conditions at the inlet (total-pressure) and the outlet (staticpressure) of the domain were established to set flow boundary conditions as neutral as possible. This enhances the quality of the numerical solutions, approaching it to the real physical process. For WMLES computations, the stochastic (nonmodeled) components at the flow boundaries are accounted for by superposing random perturbations, based on a Gaussian random function. Unfortunately, convergence history is badly-affected by this election, increasing the real time required for the model to converge (residual criterion in continuity of $10^{-6}$ ), especially at points far from design conditions. However, the numerical performance curve of the $3 \mathrm{D}$ model has presented overall good results in comparison to the performance curves of the real machine (taking into account that tip clearance has not been considered), when the working point is close to the best efficiency point (not shown here).

For every working point, $350 \mathrm{~h}$ of CPU time were necessary to obtain a periodic stable response of the model in the WMLES computations. Once the periodicity was achieved (generally after three or four rotor turns), a final running of the model was carried out to store the velocity maps during a complete blade passage (52 angular phases per rotor passage). Thus, a final post-processing, introducing similar averages to those in equations (1) and (2), provided results comparable to experimental data.

\section{NUMERICAL RESULTS}

Rotor blockage and wake shedding from both rotor and stator profiles is now observed in the numerical model. Bladeto-blade representations will illustrate wake patterns and vorticity distributions, while transversal planes will be used to compare directly both methodologies.

Blade-to-blade maps. Figure 13 shows the relative velocity distribution near the hub, normalized by the section blade speed. Nominal flow rate and $70 \% \mathrm{Q}_{\mathrm{n}}$ are introduced to show different features of flow separation and wake shedding. Obviously, the boundary layer growth is clearly evident when the flow rate is reduced. In addition, all the snapshots in the right column exhibit a displacement of the blade load towards the leading edge, conditioned by an early flow separation on the suction side. Because of that concentration near the leading edge, the aerodynamic load becomes more insensitive to the shear layer of the stator wakes. On the contrary, at nominal flow rate (left column), the impinging stator wakes are clearly modifying the shape of the blade loads. Notice the differences at $t / T_{S}=0.000$ and $t / T_{S}=0.235$ for the two blades shown in the figure. This can be also observed on the distribution of the pressure coefficient around the blades at the hub, which has been defined following typical conventions in the literature (see for instance Corsini and Rispoli, 2004, or Zhu et al., 2005,): $S=1-\left(P-P_{\text {inlet }}\right) / 0.5 \rho W_{n}^{2}$. The pressure is made non-dimensional with the relative streamwise velocity at midchord. Hence, the left plot in figure 14 shows the results at the hub, comparing the nominal distributions (grey lines) with the off-design situation (black lines). Clearly, there is a sudden increase of the pressure towards the leading edge at partial load, as already mentioned in the coloured map. The separation point is advanced to a roughly $55 \%$ of the chord length, followed by a reattachment region and a final wake shedding close to $85-90 \%$ of the blade chord. At nominal conditions, this behaviour is delayed so the blade loading presents a smoother development all over the suction side until an $80 \%$ of the blade chord. In addition, it is noticeable how at midspan (right plot of figure 14) the reduced curvature allows for much better flow conditions, moving forward the separation point for all operating conditions. 

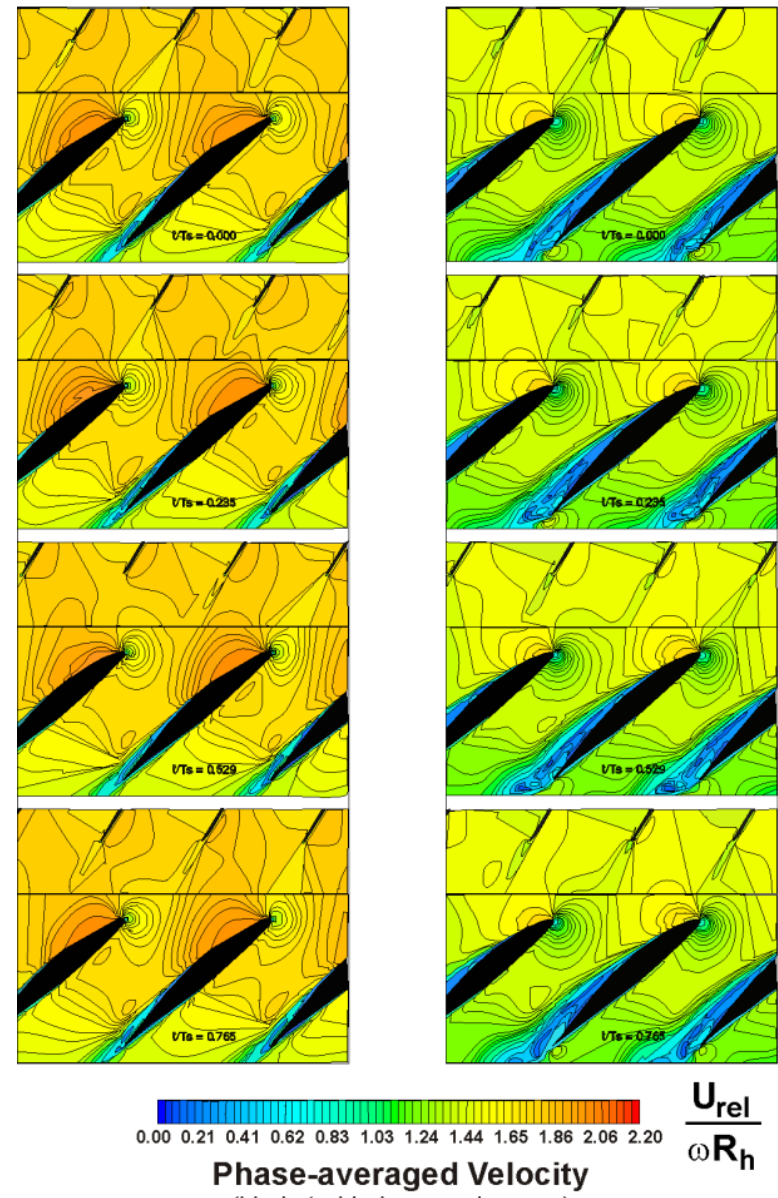

(blade-to-blade numeric maps)

Figure 13. Relative numerical velocities.

A deeper analysis of the right maps in figure 13 points that there are also major differences in the wake shedding of the rotor blades from nominal conditions to $70 \% \mathrm{Q}_{\mathrm{n}}$. Observe the evolution of the rotor wakes over time for low flow rate conditions. In that case, the WMLES computations are able to resolve the advection of large eddy structures of the flow. Precisely, previous investigations by Estevadeordal et al. (2000) pointed out the necessity for more reliable codes to capture flow separation near the fan hub, which was experimentally observed with DPIV in a similar nine-bladed fan. At $t / T_{S}=0.000$, a low-velocity structure at the trailing edge is beginning to roll-up, while at $t / T_{S}=0.529$ the eddy is already established and practically shed downstream. Since adjacent blades show the same pattern at the same instants, the frequency of these large eddies cannot be directly related to the stator wake's passing period. Otherwise, the same structures would appear in the adjacent blades but delayed in time. However, the shedding of large eddies proves to be periodic with the vane's passing frequency (in the relative frame of reference), due to the width of the shear layer. At nominal flow rate, a thinner shear layer prevents the development of any instability in the rotor wakes.
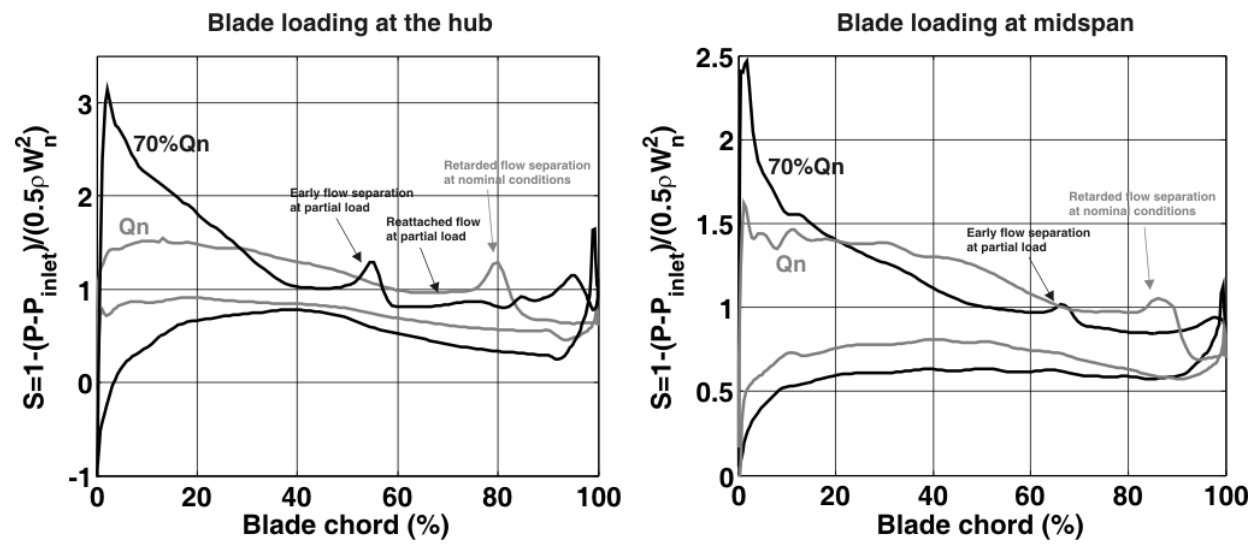

Figure 14. Numerical distributions of pressure coefficients. 
A further discussion about the unsteady effects of the stator wakes impinging on the rotor blades is introduced in figure 15. This figure shows the absolute velocity magnitude, normalized by the section blade speed, for both $37 \%$ and $50 \%$ blade chord axial gaps. At $t / T_{R}=0.00$, the stator wakes are chopping the blade loads, with a major impact in the case of the lower axial gap. Complementarily, the velocity deficit in the stator wake is periodically influenced by the stagnation point of the blades. For the lower axial gap, there is an appreciable difference between the wakes at $t / T_{R}=0.24$ and $t / T_{R}=0.76$. Thus, at rotor mid-passage locations (i.e. $t / T_{R}=0.24$ ), the wakes length decreases and its deficit is reduced (larger convection of the shear layer). On the other hand, at $t / T_{R}=0.76$, the interference is maximum, so the wakes enlarge and the velocity values decrease. This interference is also transported downstream through the rotor passage, breaking the circumferential velocity gradients between the suction and pressure sides (observable in every snapshot).

The blade-to-blade distributions are concluded with the representation of the vorticity field at the midspan. For nominal flow rate, figure 16 shows the absolute vorticity, normalized by the rotational speed. For both axial gaps, the vorticity produced by the stator wakes impinges on the blades. These lattices of wakes are then chopped by the blades and transported along the passages. In addition, the avenues of stator vorticity interface with the vorticity shed at the rotor blades, enlarging them. This effect can be observed in the differences of rotor vorticity between $t / T_{R}=0.00$ and $t / T_{R}=0.24$ for both lower and upper axial gaps. Complementarily, the rotor blockage is also revealed in the vorticity distribution at the stator. Effectively, the blades are pushing the stator wakes tangentially, as observable in the evolution of the stator wakes' orientation over time. The production of eddies at the blade's leading edge is also increased periodically due to the impact of the vorticity structures shed by the vanes. As a result, another path of vorticity is established at midpassage locations. The convection of this path with transported vorticity from the vanes leads to the generation of secondary vorticity structures, which are responsible for the enlargement and contraction of the rotor vorticity wakes.

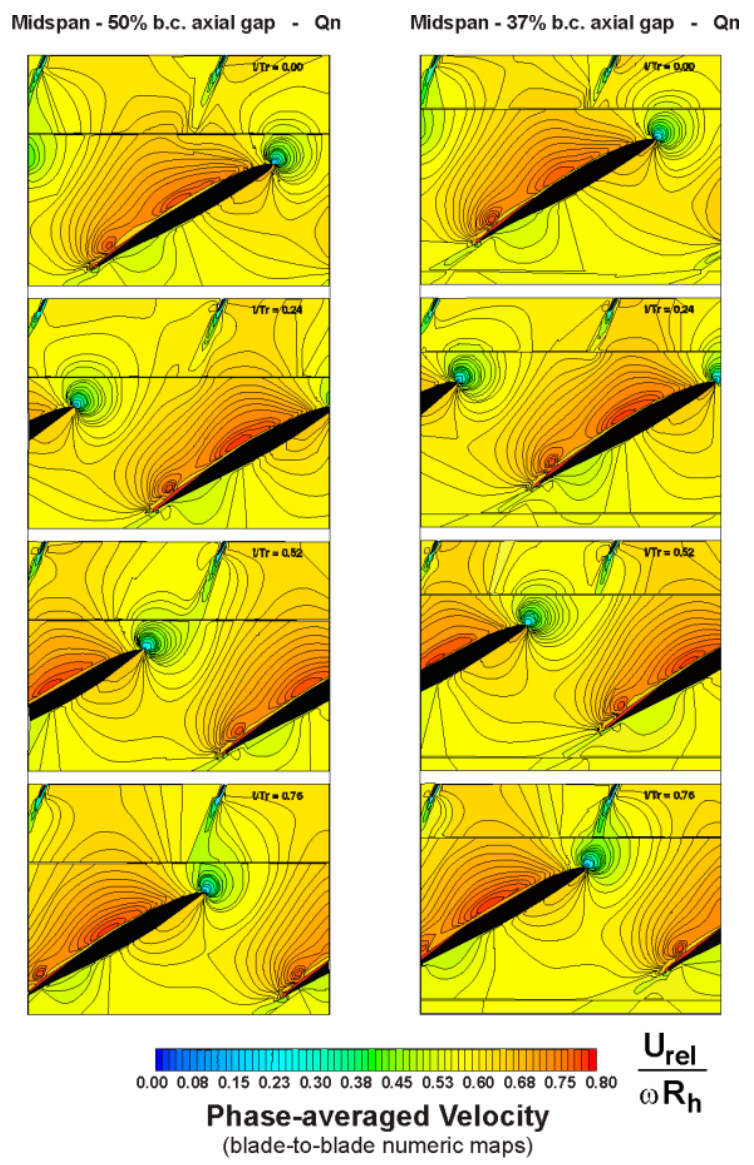

Figure 15. Numerical velocities at midspan.

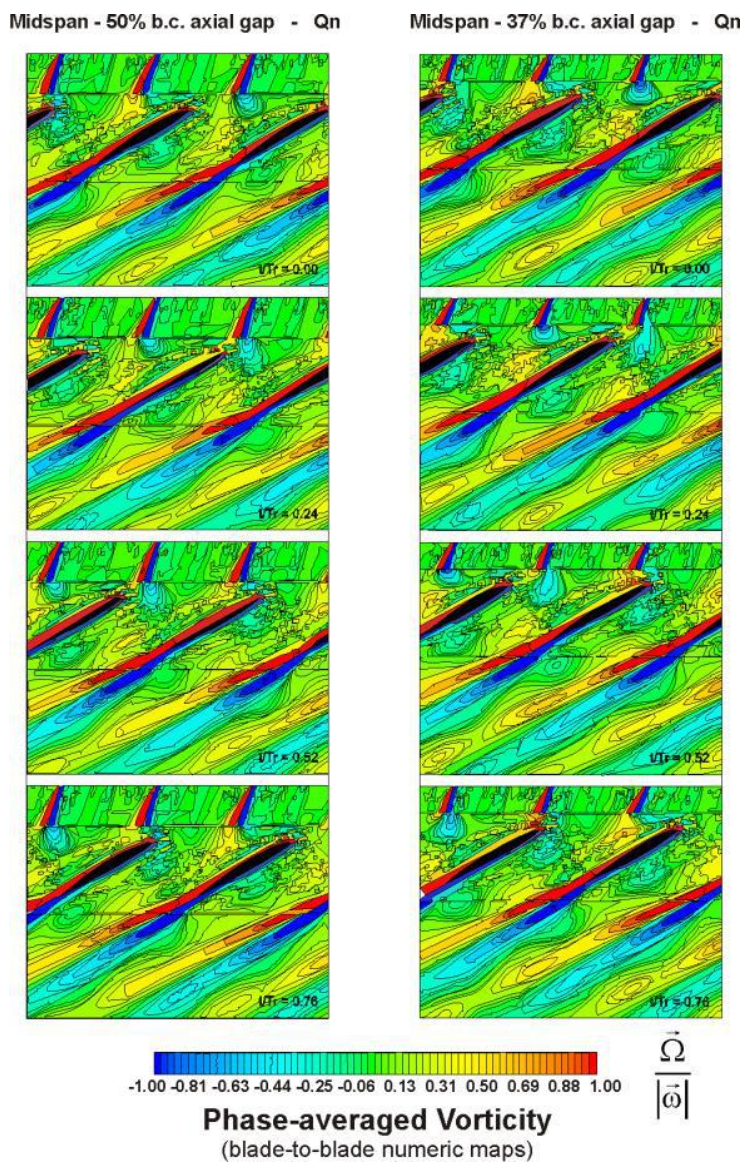

Figure 16. Numerical vorticity at midspan.

Transversal representations. Sectors analogous to those used experimentally are introduced now to illustrate the overall agreement between experiments and numerical simulations. Figure 17 shows the effect of the rotor blockage on the intensity of the stator wakes. Particularly, the influence of both axial gap distance and mass flow rate presents identical trends to those observed in the experimental data. As expected, the potential effects are much more affected by geometrical modifications (axial gap or dynamic rotation) than wake shear layers. Obviously, both gap and flow-rate reductions contribute to increasing the global unsteadiness inside the stage, because of the off-design mean flow structures.

Other rotor wakes' characteristics are included in figure 18. Relative vorticity maps were obtained in order to determine the shed boundary layer of both pressure and suction sides. From absolute velocity fields, the relative vorticity distribution was calculated according to 


$$
\frac{\left|\vec{\Omega}_{R}\right|}{|\vec{\omega}|}=\sqrt{\left(\frac{|\vec{\Omega}|}{|\vec{\omega}|}\right)^{2}+4\left(1-\frac{\operatorname{proy}_{Z} \vec{\Omega}}{|\vec{\omega}|}\right)}
$$

As expected, partial load performance shows wider rotor wakes in the left column of figure 18. Also, a clear spanwise displacement of the maximum values is related to flow rate reductions. Moreover, the wake deficit associated with the suction side is much thicker. Particularly, at $0.7 \mathrm{Q}_{\mathrm{n}}$, the wake deficit from the suction side has expanded notably onto the tip boundary layer, duplicating the size of its complementary pressure side deficit.
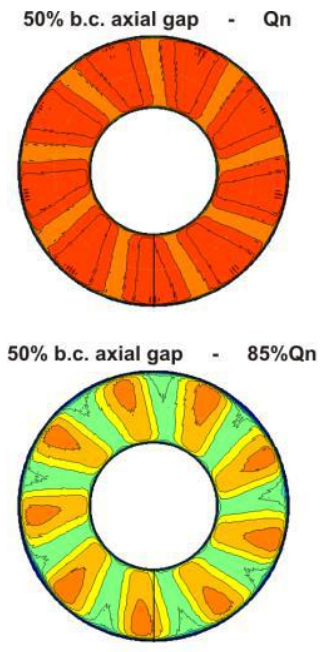

$50 \%$ b. axial gap $=-70 \% 0$

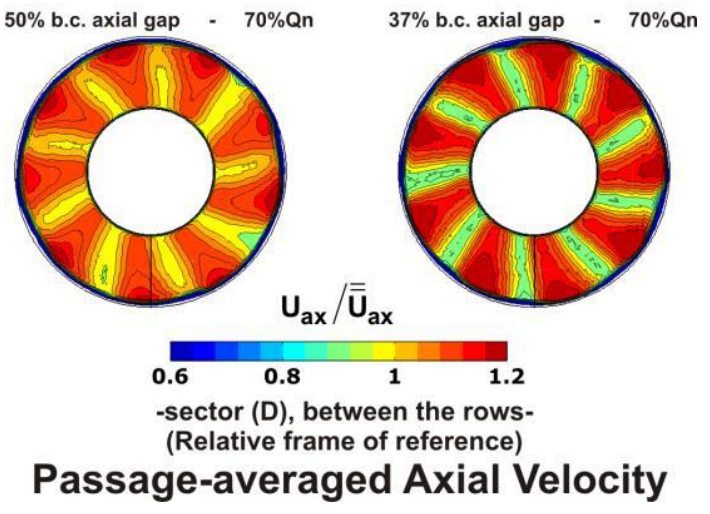

Figure 17. Full-annulus numerical velocity distributions.

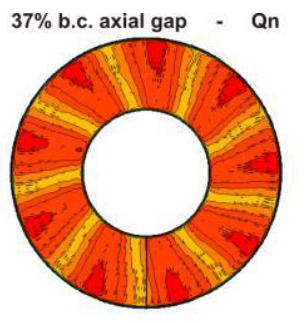

$37 \%$ b.c. axial gap - $85 \%$ Qn

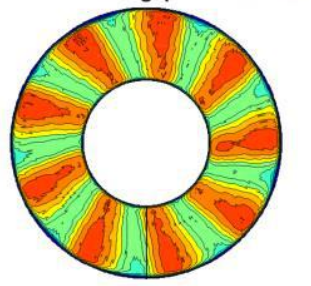

$37 \%$ b.c. axial gap $\quad-70 \%$ Qn

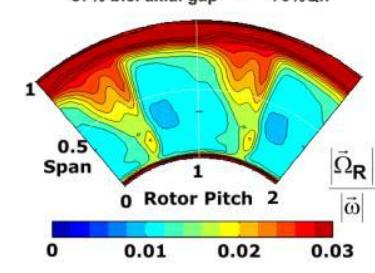

-sector (R), rotor downstream(Relative frame of reference)

\section{Passage-averaged} Relative Vorticity
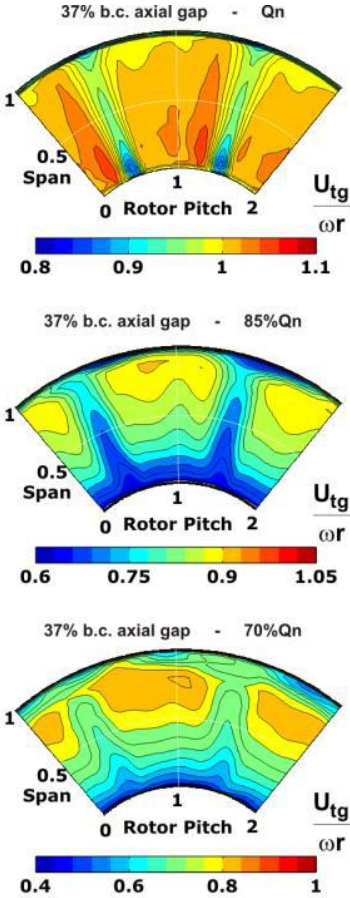

-sector ( $R)$, rotor downstream(Relative frame of reference)

Passage-averaged Tangential Velocity

Figure 18. Numerical vorticity rotor downstream.

The analysis of the rotor wake characteristics in the numerical model is completed with the right-hand column in figure 18. Relative tangential velocities are plotted, so the progressive disorder of the flow structures at the exit are evident at offdesign conditions. All cases show a more intense wake deficit at the hub section, since the radial equilibrium is completely lost.

The experimental-numerical comparison is concluded through the analysis of instantaneous velocity maps at the same plane locations $(\mathrm{D}, \mathrm{R})$. Notice the good agreement between right columns of figures 9 and 19. The rotor blockage passing effect is properly simulated in the numerical model, as well as the radial migration of the wake-rotor interaction. It is interesting to see how the blockage zone is bent backwards at the tip section $\left(t / T_{R}=0.4\right)$. In one sense, this effect is analogous to the generation of a low-velocity nodule, shown earlier in figure 7 . Therefore, and caused by the suction side of the stator vanes, the blockage passing is retarded at the tip section, so dynamic unsteadiness reaches the maximum at this location.

When rotor wakes at the moving reference frame are viewed, right column in figure 19, the unmixed-out stator wakes are also visible at the exit plane. In the figures backgrounds, the vane wakes, which are passing counter-clockwise, interact with those produced by the rotor, generating a "winding" effect on the tip section. This can be seen in the left rotor wake, comparing $t / T_{S}=0.00$ and $t / T_{S}=0.59$. Also, the numerical diffusion of the stator wakes within the rotor passages is more marked, so they have a greater width than the experimental wakes. Anyway, the overall mechanisms and the main perturbations have been captured and described by the three-dimensional numerical model. 


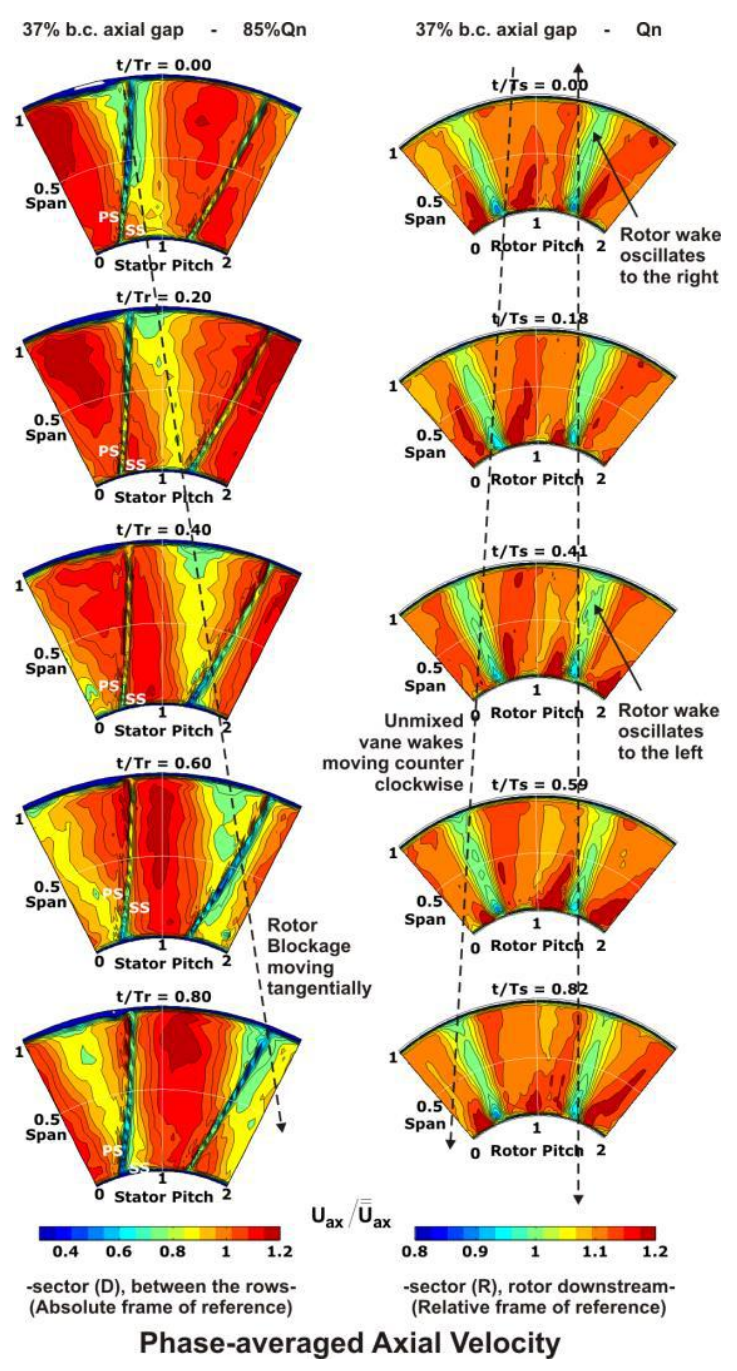

Figure 19. Numerical unsteady velocities.

Finally, the pressure distributions, at tip section, on both rotor and stator profiles have been included in figure 20 . An instantaneous realization has been represented for each one, as well as the mean distribution. At the tip, with major interaction effects as discussed above, the vane pressure profile is oscillating in such a way that even pressure and suction sides change at $t / T_{R}=0.52$ from $70 \%$ chord to trailing edge. Practically, the whole chordwise distribution is affected by the rotor blades passing downstream. On the other hand, the rotor blade loading is almost unaffected by the presence of the upstream stator, as can be seen in the coincidence of the $t / T_{S}=0.52$ instantaneous distribution and the mean.
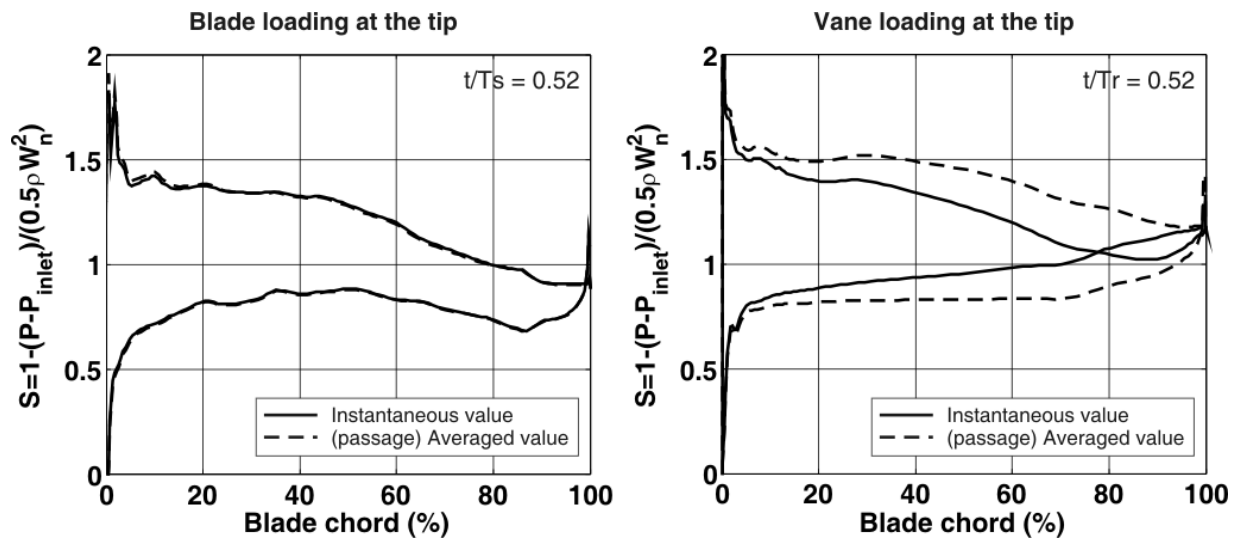

Figure 20. Unsteady loadings on vanes and blades (tip). 


\section{CONCLUSIONS}

Both experimental and numerical methodologies have been used in order to describe the interaction phenomena between fixed and rotating rows of a single stage axial flow blower. Static and dynamic features of the interaction have been analyzed; characterizing the main flow patterns in the axial gap and downstream of the rotor blades. Dual hot wire anemometry has been employed intensively to obtain complete detailed maps of the flow in the different measurement planes. In particular, axial and circumferential velocity maps were obtained within both fixed and rotating frames of reference. In a same way, an unsteady 3D numerical model of the blower has been developed. Different spatial and temporal discretizations have been explored and tested, and provide good agreement results. An LES scheme, previously contrasted with a classical RSM modelization, has also been used because of the enhancement thus achieved in the description of the wake's profile.

Rotor blade blockage has been perfectly captured in the numerical simulation, and its relation with the stator wakes' intensity has been also outlined. Experimental data showed phase dependence in the flow structures, caused by the interactions of the rows. Besides, in both methodologies the spanwise migration of increasing deficits in the stator wakes, caused by the potential effect of the rotor blockage, has been analyzed. On the other hand, the tangential velocity maps have supported similar conclusions about which locations are suffering maximum unsteady features.

This paper has also focused on the details of the wake-wake interaction. The main characteristic is the clear reminiscence of the stator wakes in the plane downwards. Obviously, the stator wakes suffer a notable decay but they are not clearly stretched or skewed by the rotor passing effect, at least at design flow conditions. The numerical modeling shows analogous behaviour, although the stator wakes seem to be more numerically diffused.

In every case, the influence of axial gap modification and off-design conditions has been included in the analysis of the wake-wake and wake-rotor characteristics. The general conclusion is that both parameters modify wake-rotor unsteady patterns, meanwhile wake-wake features are less affected by gap reductions and hardly related to partial load performance.

Future work will be focused on the realization of a deterministic analysis of the stage. The construction of the deterministic stress tensor, related to a passage-to-passage averaging technique, will identify the unsteadiness sources associated with blade row interaction, in both fixed and rotating frames of reference. This kind of tensor shows the location and intensity of the main interaction characteristics of the flow, allowing a perfect segregation of the effects of every blade row onto the total unsteady scenario of the complete stage. Finally, the steady signature of the stator wakes, still present at the exit plane, as seen above, will be related to the residual deterministic kinetic energy of the mixed-out flow, so the attenuation of stator wakes will be also studied. Therefore, the deterministic analysis claims to be a useful tool to observe and study the interaction phenomena related to multistage blade passing frequencies.

\section{NOMENCLATURE}

$\begin{array}{lll}\text { BPF } & = & \text { Blade Passing Frequency } \\ \text { DPIV } & = & \text { Digital (two-color) Particle Image Velocimetry } \\ \text { IGV } & = & \text { Inlet Guide Vane } \\ \text { LES } & = & \text { Large Eddy Simulation } \\ \text { PIV } & = & \text { Particle Image Velocimetry } \\ \text { PS } & = & \text { Pressure Side } \\ \text { RANS } & = & \text { Reynolds Averaged Navier-Stokes } \\ \text { RSM } & = & \text { Reynolds Stress Model } \\ \text { SS } & = & \text { Suction Side } \\ \text { URANS } & = & \text { Unsteady Reynolds Averaged Navier-Stokes } \\ \text { WMLES } & = & \text { Wall Modelled Large Eddy Simulation }\end{array}$

\section{Variables}

$C_{s}$

$M$

$N$

$Q, Q_{n}$

$\operatorname{Re}_{\mathrm{Ch}}$

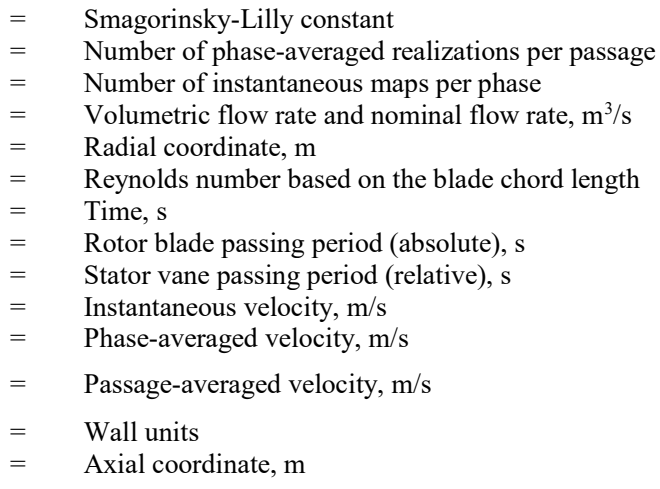

\section{Greek symbols}




$\begin{array}{lll}\theta & = & \text { Angular coordinate, deg. } \\ \phi & = & \text { Rotor phase, deg. } \\ \Omega & = & \text { Vorticity, } \mathrm{s}^{-1} \\ \Omega_{R} & = & \text { Relative vorticity, } \mathrm{s}^{-1} \\ \omega & = & \text { Rotational speed, rpm }\end{array}$

Subscripts

$\begin{array}{lll}\mathrm{ax} & = & \text { Axial component } \\ \mathrm{i} & = & \text { Velocity component } \\ \mathrm{tg} & = & \text { Tangential component }\end{array}$

\section{ACKNOWLEDGMENTS}

The authors gratefully acknowledge the finalcial support from spanish government under its "Ministerio de Ciencia e Innovación" through the research projects DPI2003-09712, DPI2006-15720 and DPI2009-13613.

\section{REFERENCES}

Adamczyk, J.J. (1985), "Model equation for simulating flows in multistage turbomachinery”, ASME paper 85-GT-226.

Argüelles Díaz, K.M., Fernández Oro, J.M., Blanco Marigorta, E. and Santolaria Morros, C. (2009), "Numerical prediction of tonal noise generation in an inlet vaned low-speed axial fan using an hybrid aeroacoustic approach". Proc. IMechE, Part C: Journal of Mechanical Engineering Science, Vol. 223, pp. 2081-2098.

Blanco Marigorta, E., Ballesteros Tajadura, R. and Santolaria Morros, C. (1998), "Angular range and uncertainty analysis of nonorthogonal crossed hot wire probes", ASME Journal of Fluids Engineering, Vol. 120, pp. 90-94.

British Standards Institution. Part 1: Methods of Testing Performance. Fans for General Purposes, BS 848 (1980).

Chow, Y-C., Uzol, O. and Katz, J. (2002), "Flow non-uniformities and turbulent hot spots due to the wake-blade and wakewake interactions in a multistage turbomachine", ASME Journal of Turbomachinery, Vol. 124, pp. 553-563.

Corsini, A. and Rispoli, F. (2004), "Flow analyses in a high-pressure axial ventilation fan with a non-linear eddy-viscosity closure", International Journal of Heat and Fluid Flow, Vol. 26, pp. 349-361.

Davidson, L. and Dahlström, S. (2005), "Hybrid LES-RANS: An approach to make LES applicable at high Reynolds numbers", International Journal of Computational Fluid Dynamics, Vol. 19 (6), pp. 415-427.

Estevadeordal, J., Gogineni, S., Copenhaver, W., Bloch, G. and Brendel, M. (2000), "Flow field in a low-speed axial fan: a DPIV investigation", Experimental Thermal and Fluid Science, Vol. 23, pp. 11-21.

Fernández Oro, J. M., Argüelles Díaz, K. M., Santolaria Morros, C. and Blanco Marigorta, E. (2007), "Unsteady flow and wake transport in a low-speed axial fan with inlet guide vanes", ASME Journal of Fluids Engineering, Vol. 129, pp. 1015-1029.

Fernández Oro, J. M., Argüelles Díaz, K. M., Santolaria Morros, C. and Ballesteros-Tajadura, R. (2009), "Impact of the tip vortex on the passage flow structures of a jet fan with symmetric blades". Proc. IMechE, Part A: J. Power and Energy, Vol. 223(A2), pp. 141-155.

Hirsch, C. (2007), Numerical computation of internal and external flows: the fundamentals of computational fluid dynamics. Ed. Elsevier-Butterworth-Heinemann.

Jang, C.-M., Sato, D. And Fukano, T. (2005), "Experimental analysis on tip leakage and wake flow in an axial flow fan according to flow rates", ASME Journal of Fluids Engineering, Vol. 127, pp. 322-329.

Lyman, F.A. (1993), "On the conservation of rothalpy in turbomachines”, ASME Journal of Turbomachinery, Vol. 115, pp. 520-526.

Piomelli, U., Van Beeck, J.P.A.J. and Benocci, C. (2006), Large eddy simulation and related techniques: theory and application. Lecture Series 2006-04. Ed. Von Kárman Institute.

Rhie, C.M., Gleixner, A.J., Spear, D.A., Fischberg, C.J. and Zacharias, R.M. (1998), "Development and application of a multistage Navier-Stokes solver: Part I - Multistage modeling using body forces and deterministic stresses", ASME Journal of Turbomachinery, Vol. 120, pp. 205-214.

Senkter, A. and Riess, W. (2000), "Experimental investigation of turbulent wake-blade interaction in axial compressors". International Journal of Heat and Fluid Flow, Vol. 21, pp. 285-290.

Sentker, A. and Riess, W. (1998), "Measurement of unsteady flow and turbulence in a low speed axial compressor". Experimental Thermal and Fluid Science, Vol. 17, pp. 124-131.

Uzol, O., Chow, Y-C., Katz, J. and Meneveau, C. (2002), "Experimental investigation of unsteady flow field within a twostage axial turbomachine using particle image velocimetry", ASME J. Turbomach., Vol. 124, pp. 542-552.

Uzol, O., Brzozowski, D., Chow, Y.-C., Katz, J. And Meneveau, C. (2007), "A database of PIV measurements within a turbomachinery stage and sample comparisons with unsteady RANS", Journal of Turbulence, Vol. 8 N10, pp. 1-25.

Van de Wall, A.G., Kadambi, J.R. and Adamczyk, J.J. (2000), "A transport model for the deterministic stresses associated with turbomachinery blade row interactions". ASME Journal of Turbomachinery, Vol. 122, pp. 593-603.

Zhu, X., Lin, W. and Du, Z. (2005), "Experimental and numerical investigation of the flow field in the tip region of an axial ventilation fan”, ASME Journal of Fluids Engineering, Vol. 127, pp. 299-307. 

This document is a pre-print version of the scientific paper published by Emerald. It has been released by the authors to fulfill all the publisher requirements established for Article Sharing:

http://www.emeraldgrouppublishing.com/authors/writing/author rights.htm\#journ al articles conditions

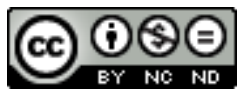

(C) 2019. This manuscript version is made available under the Creative Commons Attribution-NonCommercial-NoDerivatives 4.0 International License

(CC-BY-NC-ND 4.0 license) http://creativecommons.org/licenses/by-nc-nd/4.0/ 